\title{
Research on Virtual Disassembly and Assembly Training and Assessment System of Engine
}

\author{
Zhou Chenming ${ }^{1}$, Wang Wenju ${ }^{1+}$, Tian Yuchen ${ }^{1}$ and Jiang Zhongmin ${ }^{1}$ \\ ${ }^{1}$ University of Shanghai for Science and Technology, China
}

\begin{abstract}
In order to improve students' comprehensive understanding of the engine structure and enhance students' disassembly and assembly practice ability, this paper presents a virtual disassembly and assembly training and evaluation system for engines based on virtual reality simulation and network technology. With the help of 3dsmax modeling and Unity3D simulation program, this system builds a realistic engine disassembly and assembly scene and supports the real-time evaluation of students' experimental performance. In the system, training and evaluation functions such as experiment preview, experiment report, result management are designed, which cover all the aspects of experiment teaching. What's more, with the help of the network, students can complete the whole process of engine disassembly and assembly experiment online through the computer. The experimental results show that the system realizes the effect of virtual disassembly and assembly experiment of engine on-line, achieves the teaching purpose of classroom experiment and effectively trains the students' practical ability.
\end{abstract}

Keywords: Engine, disassembly and assembly simulation, virtual reality, internet

\section{Introduction}

At present, the traditional teaching mode of engine disassembly and assembly is usually through the teaching of slides and tools in the teacher's class. Most students lack the perceptual knowledge of the engine structure. Therefore, they are easy to lose interest in learning because they often confuse the order of experiment operation and frequently make erroneous operations. As a result, students' practice and theory are disjointed so the teaching results are not satisfactory, the traditional teaching mode can hardly meet the teaching requirements. Through virtual simulation experiments, students can interact with the computer in three-dimensional environment, so as to complete the disassembly, assembly and observation of mechanical objects and other experiments. This virtual reality simulation experimental teaching method can effectively improve students' comprehensive knowledge of the engine structure, which is a current research hotspot.

In 2011, Yuchuan Chen et al.[1] proposed a virtual laboratory based on Virtools platform, which can navigate and simulate the assembly process in multiple scenarios. However, the system can not interact with the network, nor can it realize the real-time score evaluation. In 2012, B. Shen et al.[2] proposed a Virtoolsbased education and teaching training system, which provides a virtual teaching platform for equipment maintenance training, but the system scalability is poor. In 2015, O. T. Laseinde et al.[3] proposed using VR as a tool to show engineering students some practical engineering concepts, and introduced a partial immersive virtual reality design platform developed with three-dimensional design tools. This platform enables not only conceptual learning but also remote interaction between teachers and students. In 2018, X. Yang et al.[4] proposed a virtual simulation laboratory of nursing characterized by information technology. The lab can simulate virtual training of equipment and nursing, evaluate students' nursing performance. However, the scalability of the system is not ideal. In 2019, Z. Xie et al.[5] proposed a virtual disassembly and assembly system of the front pump based on Unity3D, which realized the virtual disassembly and

\footnotetext{
+ Corresponding author. Tel.: + (86)13636675266.

E-mail address: wangwenju666@163.com.
} 
assembly process of the compressor, and provided a practical virtual disassembly and assembly system design scheme, but lacked the guidance of the experimental process and evaluation feedback.

In summary, most of the existing virtual disassembly and assembly experiment simulation systems are still on the development and research of simulation experiments. The online experimental teaching system research is not perfect, and the design and research of experimental guidance and evaluation link of experimental reports in the experimental process are still relatively insufficient. Therefore, this paper has introduced a virtual disassembly and assembly training and evaluation system for engines. This system is based on a dynamic website design, which enables students to complete experimental preview, experimental operation and submission of experimental reports online at one time, to meet the experimental teaching needs of teachers.

\section{System Function Structure}

The main function modules of the engine virtual disassembly and assembly training and evaluation system are shown in Fig. 1:

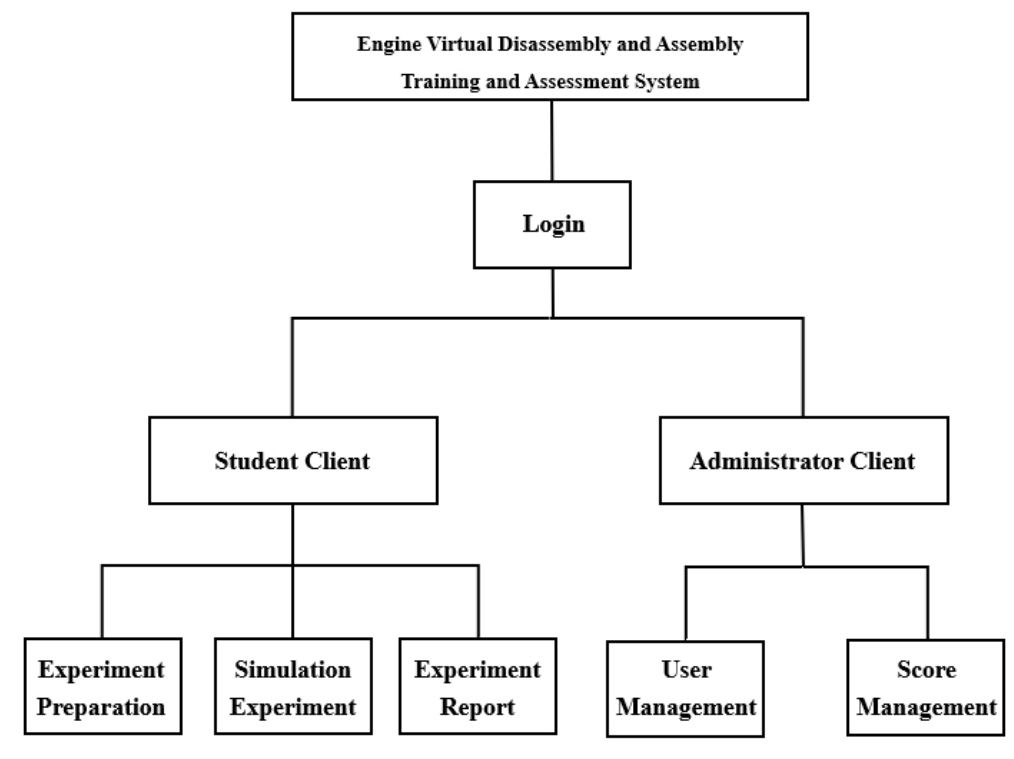

Fig. 1: System design

\subsection{Experiment Preparation}

In order to facilitate students to learn the basic knowledge of mechanical experiments, the system needs to include modules related to experimental preview, which provide students with the functions of reading and downloading electronic documents. The electronic documents should be compiled with reference to the relevant experimental operation guide of the school laboratory and the experimental operation design of virtual simulation experiments. In this module, students can preview the experiment and get some key information for the experiment process.

In order to improve the compatibility of the system with computers, the system mainly uses PDF format electronic documents. The PDF format file is easy to transfer and store, supports encapsulation of information such as fonts, formats, colors, graphics and images, does not depend on the language and fonts of the computer operating system and display devices. Therefore, the PDF format file is suitable for use in dynamic website systems.

\subsection{Virtual Experiment Operation}

In the aircraft engine disassembly and assembly simulation experiment, students can complete the disassembly and assembly of the engine through keyboard and mouse operation, while supporting the movement and scaling of parts. The system automatically completes the evaluation of students' experimental performance in the course of the experiment. The experimental design is as follows: 
(1) Engine disassembly operation: Each module of the engine can be separately shifted and dispersed to a set coordinate position by the keyboard and mouse action of the user. Due to the requirement of a certain sequence of engine disassembly, the system can record the user's disassembly operation sequence and prompt them when the operation is wrong until the user succeeds.

(2) Engine assembly operation: By selecting the mouse and triggering the keyboard keys, the parts can be assembled to the initial position, and the system can record the order of user's assemble operation, and will also prompt for error operation information until the operation is successful. The experiment is completed when the user has finished assembling the whole engine.

(3) Evaluation of experimental procedures: The engine is divided into several modules according to the structure and function of the engine, and a certain weight of operation score is set according to the size and importance of the modules. In the disassembly and assembly process, the operation of each module will be automatically judged by the score. From the second operation error, the system will automatically give a certain penalty based on the weight of the module and the number of operation errors. When the operation is successful, it will also give a certain score reward according to the number of times required for success.

\subsection{Experiment Report}

The experiment report is the important feedback of students to the experiment experience, and it is also one of the important references for teachers to score students' performance. According to the content requirements of laboratory experiment report, six parts are designed under the module of experiment report: experiment purpose and task, experiment principle and knowledge point, experiment process and main content record, experiment result, conclusion and problem discussion, thought and suggestion. Students can fill in the corresponding content of each section online. After clicking the submit button, the website should generate the experimental report information and store it in the database. Teachers can view and evaluate the experimental report content through the performance management module of the administrator side website, and then upload the experimental report database to store the score information through the website. Finally, students can log in to view their own ratings.

\section{System Function Realization}

The main function implementation of this system is divided into three major parts: experimental preview function implementation; virtual experiment operation function implementation; experimental report evaluation function implementation.

\subsection{Experimental Preview Function Implementation}

The experiment preview function has undergone three major processes:

(1) Through the guidance of the school experimental operation guide book and the opinions of relevant professional teachers and experimenters. The system developer has organized and compiled a lab operation specification and a word file of the experimental guidance book combined with the operation set by the developed experimental program.

(2) Call the word printing function to use the PDF printer to convert the file to PDF format. In the system, the system designed a special folder for storing experimental preview files, and put the generated PDF file into the folder.

(3) In the experiment preview module page, the storage address of PDF file is retrieved through the web page, and the PDF display control is loaded to display the PDF content. At the same time, it supports PDF document enlargement, full screen view, download, printer printing and other operations.

\subsection{Virtual Experiment Operation Function Implementation}

The realization of the operation function of virtual simulation experiment mainly experiences two processes: three-dimensional modeling and virtual simulation disassembly and assembly instance simulation.

(1) The modeling process steps are as follows: 
(1) Get the design drawings of the aircraft engine for the parts information of the flight engine, and then select the appropriate scale to model. Considering the rendering efficiency and the running effect of Unity3D environment, the model size of this paper is 1:100.

(2) Three-dimensional modeling tools are used to model each module of the aircraft engine. The basic features of the model are created by using arrays, stretching commands, and then the shape of the model is modified by chamfering, drawing, etc. Finally, the relative positions of parts and parts are continuously adjusted to complete the creation of each module of the model.

(3) The modules are grouped into a complete aircraft engine model, the model file in FBX format is exported using 3dsmax to stand by. Finally, the model file is dragged into the created Unity project and imported into the environment.

(2) The steps to develop virtual simulation experiments are as follows:

(1) Engine Parts Split Classification: Twelve empty objects are created for 12 different parts of the engine. After renaming, the corresponding parts are dragged into the corresponding empty objects to classify the model parts and give different material effects to the different parts.

(2) Determination of disassembly and assembly position and moving effect of engine parts: First, in order to accurately determine the position of the parts after moving, the developer adds a fixed point object to each part in the unit program, using the coordinates of each point as the position after moving. A script was written to store the initial and moved positions of each part of the engine, to achieve the moving effect of the parts during disassembly, and to record the fractions at the same time. The engine assembly process is the reverse of the disassembly process described above.

(3) Instance Program Publishing: After verifying that the virtual emulator is working correctly, select the WebGL option in the Building Setting under File to publish to a folder containing an HTML display page. Import the generated folder into the dynamic website project file, and use the display page to load the aircraft engine disassembly and assembly simulation experiment, complete the development of virtual experiment operation module.

\subsection{Experimental Report Evaluation Function Implementation}

(1) Each module of the experiment report creates a corresponding text box for students to fill in. Students can click on the button to submit a report after they have completed the six major parts of the experimental report: the purpose and task of the experiment, the principles and points of knowledge of the experiment, the process and main contents of the experiment, the results of the experiment, conclusions and questions, thoughts and suggestions. The website automatically uploads the corresponding data to the database, and the page returns the prompt for successful submission when the database storage is complete.

(2) Through the administrator side, the teacher requests the submitted experimental report data from the database in the report scoring module, and displays the experimental report content in the text box. Teachers can enter scores into another text box after viewing them, and after confirmation, the website automatically uploads the score data to the database.

(3) Students can query their final scores through the experimental results query module. A table is designed to request and display data of school number, name, report type, score and teacher number. Students can easily query related information.

\section{Experimental Results and Analysis}

This system is based on WIN10 operating system and developed with VS2015 and Microsoft SQL Server 2008 R2 database. To test the effectiveness of the system, the developer simulated the engine virtual disassembly and assembly training experimental process as shown in Figures 2 to 6 .

Before logging into the system, a new student account is registered through the administrator account for the operator to use. When an operator enters the site through a browser, the operator will enter the home page of the site, as shown in Figure 2. Operators can enter the student or administrator side by entering the school number or work number and password on this page and entering the authentication code after selecting the 
user type for login operation. Since the operator's privilege is student privilege, the operator will log on to the system student side.

After logging in to the student client, the operator can preview the experimental specifications and methods of operation by choosing "Experimental Overview - Lab Operational Specifications" or "Experimental Overview - Experimental Instructions" in the left guide bar, as shown in Figure 3.

After previewing the experiment, the operator can then select "Simulation Experiment - Airplane Engine" from the left guide bar of the system to enter the simulation experiment page for simulation experiment. Figures 4 and 5 show that the operator can clearly observe and operate the assembly and disassembly of the 3D engine (mesh model or 3D point cloud).(This sample experiment program is developed with reference to the sample program of virtual simulation and game development practical tutorial[6]) Figure 4 shows the after-assembly configuration of the engine, and Figure 5 shows the parts after disassembly of the engine.

After the experiment is completed, the operator can select "Experimental Report-Submit Report" in the left guide bar of the system to enter the experimental report writing page, as shown in Figure 6. After choosing the appropriate kind of experiment, the operator can fill in the contents of the experiment report in the text box, and then click the button to submit. Operators can then confirm what they have completed in the "Experimental Report - View Report" on the left guide bar. After the teacher has scored the experiment report, the operator can select "View results-experiment results query" in the left guide bar to enter the results query page and see their own experiment results information.

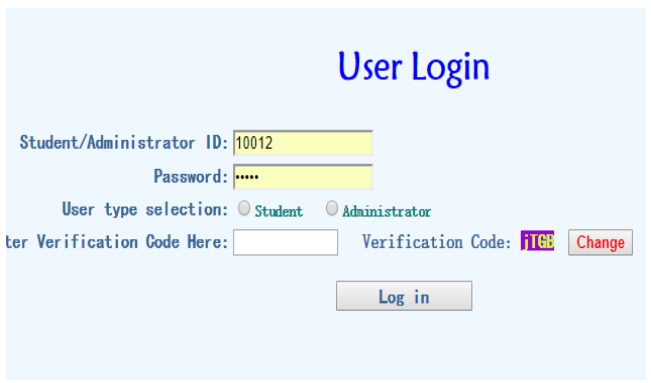

Fig. 2: System home page

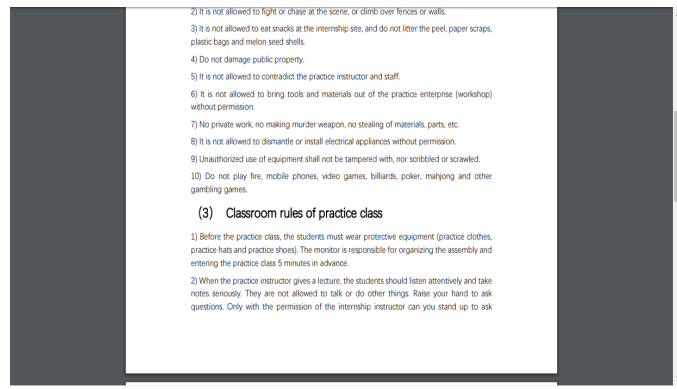

Fig. 3: Experimental preview
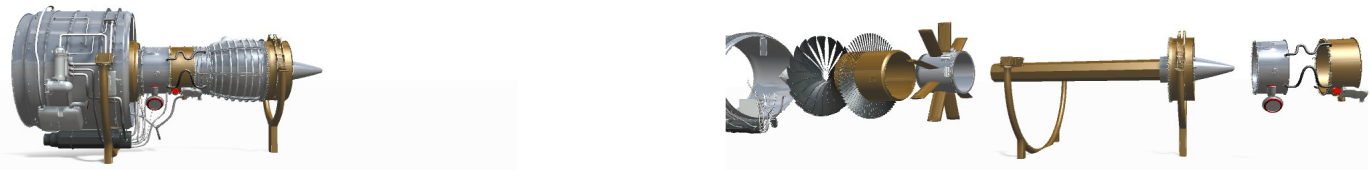

Fig. 4: Airplane engine

Fig. 5: Airplane engine parts

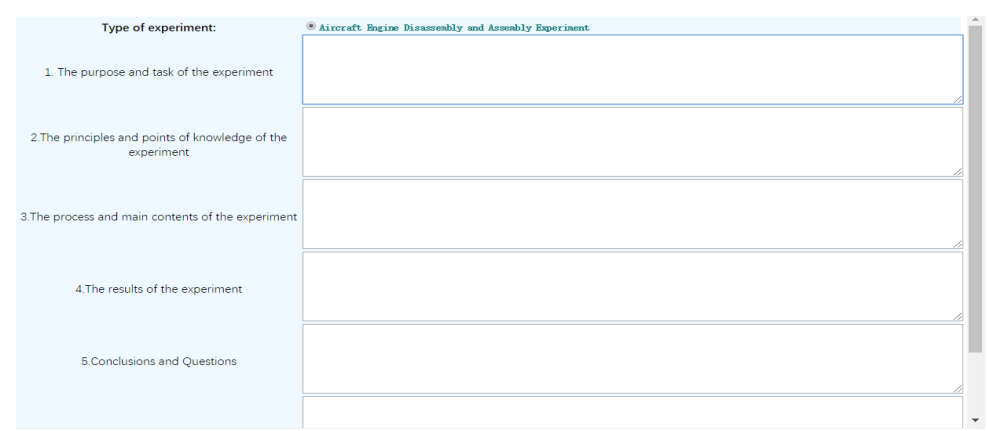

Fig. 6: Experimental report writing 
Through the legend and operation description above, this system can realize the functions of experiment preview, experiment operation, submission of experiment report, and provide an effective training and evaluation system for the virtual disassembly and assembly of engines for students and teachers.

\section{Conclusion}

This paper introduces an engine virtual disassembly and assembly training and assessment system, which is based on Unity3D, dynamic website, database and other technologies. This system achieves many functions such as displaying experimental guide files, carrying out simulation experiments for engine disassembly and assembly, composing experimental reports online and submitting reports. Users can perform simulation experiments of engine disassembly and submit job reports according to the instructional requirements through this system. Teachers can also use this system to conduct experimental teaching for students so that students can quickly and efficiently understand and master the engine structure and experience the experimental process. Thus, the system provides users with a vivid teaching environment, constructs a new experimental teaching mode for teachers, and improves the efficiency of experimental teaching as a whole. This system can not only be applied to engine virtual disassembly and assembly, but also support the expansion of other Unity3d virtual simulation experiment programs, which can provide reference for the research and development of other mechanical simulation experimental platforms.

\section{Acknowledgements}

The financial support for this work was provided in part by the Natural Science Foundation of Shanghai under Grant 19ZR1435900, Virtual reality technology foundation-2019 quality online course cultivation project of University of Shanghai for Science and Technology

\section{References}

[1] Yuchuan Chen, Qingni Hu and Hong Shu, "A virtual lab for mechanical experiment based on virtools," 2011 IEEE International Conference on Computer Science and Automation Engineering, Shanghai, 2011, pp. 119-122.

[2] B. Shen, Z. Tang, T. Wang and J. Zhao, "Equipment Training Teaching Based on VIRTOOLS," 2012 4th International Conference on Intelligent Human-Machine Systems and Cybernetics, Nanchang, Jiangxi, 2012, pp. 126-129.

[3] O. T. Laseinde, S. B. Adejuyigbe, K. Mpofu and H. M. Campbell, "Educating tomorrows engineers: Reinforcing engineering concepts through Virtual Reality (VR) teaching aid," 2015 IEEE International Conference on Industrial Engineering and Engineering Management (IEEM), Singapore, 2015, pp. 1485-1489.

[4] X. Yang, Y. Li, L. Wang and D. Li, "Development and Application of Nursing Operation Virtual Simulation Training System," 2018 International Conference on Engineering Simulation and Intelligent Control (ESAIC), Changsha, 2018, pp. 185-187.

[5] Z. Xie, Y. Zhou, Y. He and X. Fu, "Design of Virtual Disassembly System of Front Pump Based on Unity-3d," 2019 International Conference on Virtual Reality and Intelligent Systems (ICVRIS), Jishou, China, 2019, pp. 1719.

[6] Unity Technologies, "Virtual simulation and game development practical tutorial"[CP/DK]. Shanghai: Shanghai Jiao Tong University Press,2015. 